TITLE:

\title{
Adsorption and order formation of colloidal nanoparticles on a substrate: A Brownian dynamics study
}

\section{AUTHOR(S):}

Miyahara, M; Watanabe, S; Gotoh, Y; Higashitani, K

\section{CITATION:}

Miyahara, M ...[et al]. Adsorption and order formation of colloidal nanoparticles on a substrate: A Brownian dynamics study. JOURNAL OF CHEMICAL PHYSICS 2004, 120(3): 1524-1534

\section{ISSUE DATE:}

2004-01-15

URL:

http://hdl.handle.net/2433/39755

\section{RIGHT:}

Copyright 2004 American Institute of Physics. This article may be downloaded for personal use only. Any other use requires prior permission of the author and the American Institute of Physics. 


\title{
Adsorption and order formation of colloidal nanoparticles on a substrate: A Brownian dynamics study
}

\author{
Minoru Miyahara, ${ }^{\text {a) }}$ Satoshi Watanabe, Yoshiaki Gotoh, and Ko Higashitani \\ Department of Chemical Engineering, Kyoto University-Katsura, Nishikyo-ku, Kyoto, 615-8510 Japan
}

(Received 3 March 2003; accepted 20 October 2003)

\begin{abstract}
Adsorption process and order formation of electrostatically stabilized colloidal particles with a radius of $50 \mathrm{~nm}$ onto a planar surface with countercharge are examined. We perform Brownian dynamics simulations with a new three-dimensional cell model, in which the particle-particle and particle-substrate interactions are modeled based on the DLVO theory. The simulations yield the following results: (1) a larger bulk concentration would be required for larger $\kappa a$ to reach order formation to compensate for the decrease in the bulk potential; (2) the phase transition from a disordered to an ordered structure of the adsorbed particles on the substrate is considered to be of the Kirkwood-Alder type of transition through the examination of the two-dimensional pressure of the adsorbed particles; (3) the adsorbed particles are found to form a hexagonally ordered array, only if what we call "one-directional average force" acting on an adsorbed particle exceeds a critical value, which is independent of the ionic strength, or the interaction potentials. The critical value of the one-directional average force is interpreted as the force needed to keep an ordered structure by localizing adsorbed particles at fixed positions. In addition, the critical force is used to develop a new model to estimate the surface coverage at the order-disorder transition and it is demonstrated that the new model gives better estimation than other models previously reported. (C) 2004 American Institute of Physics. [DOI: 10.1063/1.1632896]
\end{abstract}

\section{INTRODUCTION}

Ordered arrays of particles on a substrate have attracted increased attention recently because of their unique functions especially in optical properties, some examples of which include antireflection coating, ${ }^{1}$ data storage, a new sensor, and a template for photonic crystals. ${ }^{2}$ The important particle size would be from submicron down to nanometers, or the nanoparticles in the broad sense. It is of crucial importance to have them self-assembled from the viewpoint of industrial manufacturing. Although the assembly of particles can be made by the Langmuir-Blodgett technique ${ }^{3}$ and the convective assembly method, ${ }^{4}$ these methods may be accompanied by difficulties in handling materials or limitation of particle size. The adsorption of colloidal particles on a flat substrate and their spontaneous ordering would thus be a first possibility.

In general, the key factor for making up an ordered structure would be to make the density of elemental particles be high enough so that repulsive characteristics of the elements would appear. The freezing of simple fluid upon cooling or pressurizing is a typical example: the molecules form crystal phase with, e.g., fcc structure when the density exceeds a certain critical value. Thus a strategy for establishing two-dimensional order for colloidal system would first be the introduction of an adsorptive substrate: if the attraction of the substrate can be sufficient to make the surface coverage high enough, the adsorbed particles must make up an ordered array which can accommodate a larger number of particles.

\footnotetext{
a) Author to whom correspondence should be addressed. Electronic mail: miyahara@cheme.kyoto-u.ac.jp
}

Electrostatic attraction by a countercharged surface is thought to be applicable to electrostatically stabilized colloidal particles.

Experimentally, the direct observation of structures of adsorbed nanoparticles on a substrate has been done with the scanning probe microscope (SPM), the atomic force microscope (AFM), and the scanning electron microscope (SEM). Johnson and Lenhoff ${ }^{5}$ observed structures of positively charged latex particles dispersed on a mica substrate with AFM in air and found a high degree of short-range order among adsorbed particles in their experiments studying the effect of electrolyte concentration on adsorption kinetics and the ordering of adsorbed particles. Semmler et al. ${ }^{6,7}$ also showed the AFM images in which adsorbed latex particles were uniformly distributed over a mica substrate at low electrolyte concentration through their experiments examining the effect of electrostatic interaction and particle size polydispersity on adsorption process and surface coverage. Antelmi and Spalla, ${ }^{8}$ using SPM, examined the possibility of the lateral migration of adsorbed latex particles on a sapphire substrate and attributed two-dimensional aggregation mainly to the migration of the particles along the substrate. Yamaguchi et al. ${ }^{9,10}$ fabricated a latex-particle monolayer with relatively regular intervals on a glass or alkylated glass plate observed with SEM. Though no detailed analysis has been reported on the structure on a substrate, these results mentioned above would be sufficient to show the possibility of the formation of an ordered structure.

On the other hand, computer simulation is a useful tool to analyze the behavior of particle adsorption onto a substrate. The random sequential adsorption (RSA) model, ${ }^{11}$ fol- 
lowed by some improvements, ${ }^{12-19}$ has been mainly used to describe the long-time adsorption behavior of colloidal particles onto a strongly attractive substrate, and to predict the maximum surface coverage, known as the jamming limit. The RSA model is, however, a two-dimensional model, which includes neither the effect of particle concentration in bulk nor surface diffusion of adsorbed particles. Due to the lack of these factors, two-dimensional models are not suitable to deal with order formation. Therefore, a threedimensional model is strongly required for this purpose. Few of the three-dimensional models, however, have been proposed so far because of the difficulty in treating the boundary condition of a simulation cell with an adsorptive surface: the periodic boundary condition cannot be imposed for the direction normal to the surface.

Oberholzer et $a ._{.}{ }^{20}$ and Gray and Bonnecaze ${ }^{21}$ have dealt with this problem in their respective different approaches to keep the bulk chemical potential constant. The former has developed a technique incorporating a grand canonical Monte Carlo reservoir and a Brownian dynamics cell, examining the effect of salt concentration on adsorption kinetics and surface coverage for polystyrene latex particles and a protein lysozyme, though the structure of adsorbed particles was not examined. The latter carried out Brownian dynamics simulations with an open boundary cell considering a meanfield force due to particles in the bulk acting on particles in the simulation cell, and studied systematically the effect of particle potentials, wall potentials, volume fractions, and salt concentration on order-disorder boundaries of the adsorbed phase in addition to the effect of them onto short-time kinetics, long-time kinetics, and surface coverage. Further, they conducted simulations $^{22}$ of the system with charge-bidisperse mixtures of particles. An extensive analysis of the dependence of wall potential and particle potential on orderdisorder transition was done in the above study, but the mechanism of order formation still remains to be elucidated.

Our main objective is to clarify the mechanism of order formation and to develop a model to describe the rate process of the order formation. As the first step to our objective, we propose a new three-dimensional model to simulate the adsorption of colloidal particles onto an adsorptive surface under a specified bulk concentration, based on the Brownian dynamics technique. The adsorption process of electrostatically stabilized colloidal particles with a radius of $50 \mathrm{~nm}$ onto a planar surface with countercharge is simulated with the particle-particle and particle-surface interactions modeled on the DLVO theory. ${ }^{23}$ The detailed structure of the adsorbed phase is examined to find out the key factor for establishing an ordered state.

\section{MODEL AND METHOD}

\section{A. Brownian dynamics}

A direct simulation of colloidal dispersion by molecular dynamics would be practically impossible because of the large number of the solvent molecules, which leads to a great increase in the computational cost. Brownian dynamics method, devised by Ermak, ${ }^{24}$ treats only solute particles, regarding the solvent as a continuum medium and representing the thermal effect of the solvent by random Brownian forces acting on the solute particles; it is suitable for the simulations of the colloidal dispersion.

The motion of a particle $i$ including the Brownian motion is represented by the Langevin equation:

$$
m_{i} \frac{\mathrm{d} \mathbf{v}_{i}}{\mathrm{~d} t}=\mathbf{F}_{i}^{\mathrm{P}}-\xi \mathbf{v}_{i}+\mathbf{F}_{i}^{\mathrm{B}},
$$

where $m_{i}$ is the particle mass, $\mathbf{v}_{i}$ is the velocity vector, $\mathbf{F}_{i}^{\mathrm{P}}$ is the external force due to the particle-particle and particlesubstrate potentials, which is modeled based on the DLVO theory, as explained later, $\xi \mathbf{v}_{i}$ is the hydrodynamic drag force, and $\mathbf{F}_{i}^{\mathrm{B}}$ is the random force causing the Brownian motion. The friction factor $\xi$ is given by Stokes' law,

$$
\xi=6 \pi \eta a,
$$

where $\eta$ is the solvent viscosity and $a$ is the radius of the colloidal particle. No hydrodynamic interactions between particles are considered in the present study because particles keep apart from each other due to the repulsive double-layer force.

When the size of the solute particle is much larger than that of the solvent molecule, which applies to most of the colloidal dispersions, Eq. (1) can be expressed by the following discrete form: ${ }^{24}$

$$
\mathbf{r}_{i}(t+\Delta t)=\mathbf{r}_{i}(t)+D \frac{\mathbf{F}_{i}^{\mathrm{P}}}{k_{\mathrm{B}} T} \Delta t+\Delta \mathbf{r}_{i}^{\mathrm{B}},
$$

where $\mathbf{r}_{i}(t)$ is the position vector at time $t, \Delta t$ is a time step, $k_{\mathrm{B}}$ is the Boltzmann constant, $T$ is the temperature, and $D$ is the diffusion coefficient given by the Stokes-Einstein relation, $D=k_{\mathrm{B}} T / 6 \pi \eta a$. The random displacement $\Delta \mathbf{r}_{i}^{\mathrm{B}}$ caused by the random force $\mathbf{F}_{i}^{\mathrm{B}}$ forms the Gaussian distribution of the following character:

$$
\left\langle\Delta \mathbf{r}_{i}^{\mathrm{B}}(t)\right\rangle=\mathbf{0}, \quad\left\langle\left(\Delta r_{i, k}^{\mathrm{B}}(t)\right)\left(\Delta r_{j, l}^{\mathrm{B}}(t)\right)\right\rangle=2 D \Delta t \delta_{i j} \delta_{k l},
$$

where $k$ and $l$ are indices for the coordinate directions $(x, y, z)$, and $\delta_{i j}$ is the Kronecker delta function.

\section{B. DLVO potential}

The external force $\mathbf{F}_{i}^{\mathrm{P}}$ for suspension particles with surface charge can be calculated from the particle-particle and particle-substrate potentials based on the DLVO theory, ${ }^{23}$ which is composed of the electrostatic and the van der Waals interactions.

The electrostatic potential is given by the following equations $^{20,25}$ scaled by $k_{\mathrm{B}} T$ :

$$
\begin{aligned}
E_{\mathrm{el}(\mathrm{pp})}= & \left(\frac{4 \pi k_{\mathrm{B}} T \epsilon \epsilon_{0} a}{e^{2}}\right)\left(\frac{\psi_{\mathrm{p}}+4 \gamma \Omega \kappa a}{1+\Omega \kappa a}\right)^{2} \frac{1}{r} \\
& \times \exp [-\kappa a(r-2)], \\
E_{\mathrm{el}(\mathrm{ps})}= & \left(\frac{4 \pi k_{\mathrm{B}} T \epsilon \epsilon_{0} a}{e^{2}}\right)\left(\frac{\psi_{\mathrm{p}}+4 \gamma \Omega \kappa a}{1+\Omega \kappa a}\right) \\
& \times\left[4 \tanh \left(\frac{\psi_{\mathrm{s}}}{4}\right)\right] \exp [-\kappa a h],
\end{aligned}
$$




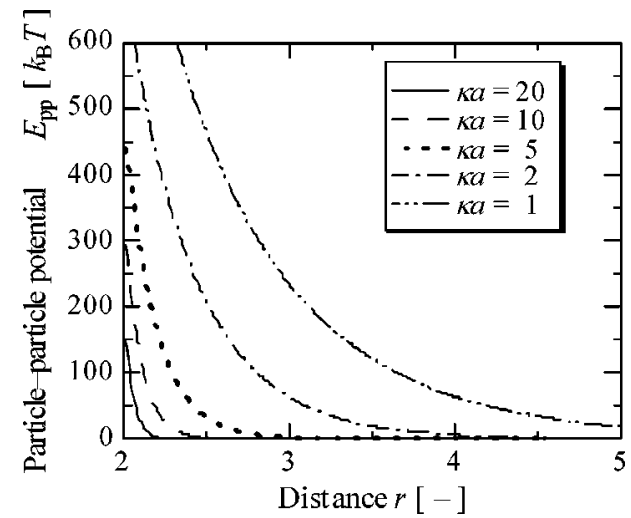

FIG. 1. Potential curves of particle-particle interactions for five values of $\kappa a$.

where $E_{\mathrm{el}(\mathrm{pp})}$ is the particle-particle electrostatic potential, $E_{\mathrm{el}(\mathrm{ps})}$ is the particle-substrate electrostatic potential, and $r$ and $h$ are the distance between particle centers and the distance between the substrate and the particle surface, respectively, both of which are scaled by the radius $a . \epsilon$ is the relative permittivity of the solvent, $\epsilon_{0}$ is the vacuum permittivity, $e$ is the elementary charge, $\kappa$ is the inverse Debye length, and $\psi_{\mathrm{s}}$ and $\psi_{\mathrm{p}}$ are the electrical potentials of the substrate and the particle, respectively: both the potentials are scaled by $k_{\mathrm{B}} T / e$. $\gamma$ and $\Omega$ are given by

$$
\gamma=\tanh \left(\frac{\psi_{\mathrm{p}}}{4}\right) \quad \text { and } \Omega=\frac{\psi_{\mathrm{p}}-4 \gamma}{2 \gamma^{3}} .
$$

The van der Waals potential is expressed by the dimensionless form with the scaling factor $k_{\mathrm{B}} T$,

$$
\begin{aligned}
& E_{\mathrm{vdW}(\mathrm{pp})}=-\frac{A_{\mathrm{pp}}}{6 k_{\mathrm{B}} T}\left[\frac{2}{r^{2}-4}+\frac{2}{r^{2}}+\ln \left(1-\frac{4}{r^{2}}\right)\right], \\
& E_{\mathrm{vdW}(\mathrm{ps})}=-\frac{A_{\mathrm{ps}}}{6 k_{\mathrm{B}} T}\left[\frac{1}{h}+\frac{1}{h+2}+\ln \left(\frac{h}{h+2}\right)\right],
\end{aligned}
$$

where $E_{\mathrm{vdW}(\mathrm{pp})}$ is the van der Waals potential between two particles, $E_{\mathrm{vdW}(\mathrm{ps})}$ is the van der Waals potential between a particle and a substrate, and $A_{\mathrm{pp}}$ and $A_{\mathrm{ps}}$ are Hamaker's constants between two particles and between a particle and a substrate, respectively.

The particle-particle potential curve is shown in Fig. 1 and the particle-substrate potential curve in Fig. 2. The particle-particle interaction is repulsive and the particlesubstrate interaction is attractive under the condition employed in the present study. Here $\kappa a$ is the ratio of the radius of the particles to the thickness of the electric double layer, which determines the range of the electrostatic interaction and is related to the salt concentration $C$ through the following equation:

$$
\kappa^{-1}=\frac{0.304}{\sqrt{C}} \mathrm{~nm},
$$

at $25^{\circ} \mathrm{C}$ for a $1: 1$ electrolyte.

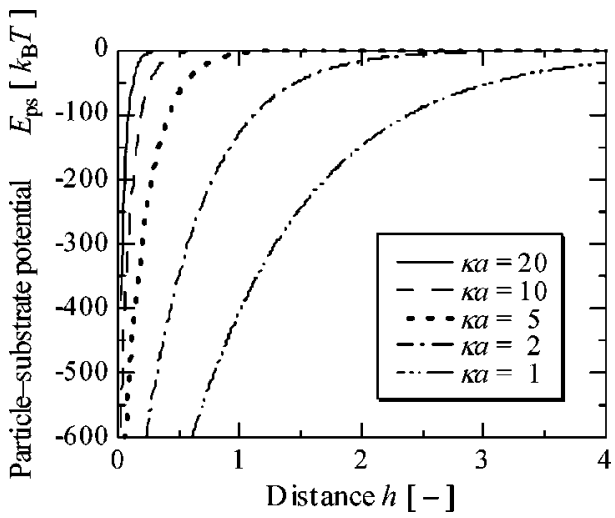

FIG. 2. Potential curves of particle-substrate interactions for five values of $\kappa a$.

\section{Simulation model}

The simulation cell used is composed of two parts, as illustrated in Fig. 3: the main cell and the virtual bulk cell. As for the main cell, a flat substrate of uniform electric charge opposite to that of the particles is set at the bottom of the main cell, and the colloidal particles adsorb onto the substrate because of the electrostatic interaction between the particles and the substrate. The nearest distance between the particle surface and the substrate is assumed to be $0.4 \mathrm{~nm}$ because of the steric exclusion. The equation of motion of adsorbed particles is assumed to be the same as that of bulk particles. However, the interaction between particles and the substrate is so strong under the condition employed in the present study that the particle adsorption is efficiently irreversible, as described in Sec. III A. Consequently, the motion of particles on the substrate is substantially confined to $x, y$ directions. At the top of the main cell, the particles do not come across the boundary to the virtual bulk cell, but reflect back into the main cell. Periodic boundary conditions are applied in the $x$ and $y$ directions. A reservoir region, which maintains the desired particle concentration, is set in the upper part of the main cell. In order not to have the decrease in

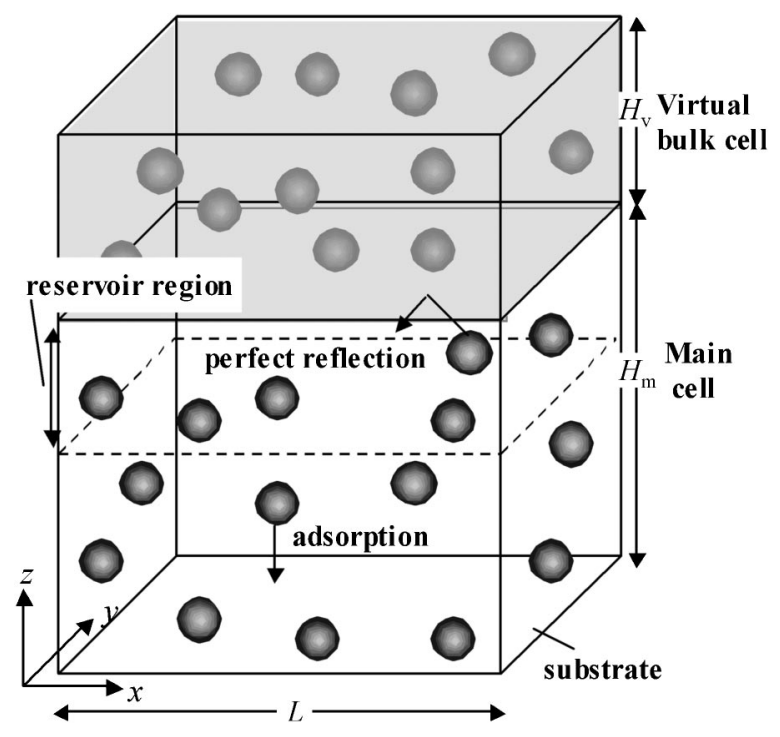

FIG. 3. A schematic drawing of the simulation cell. 


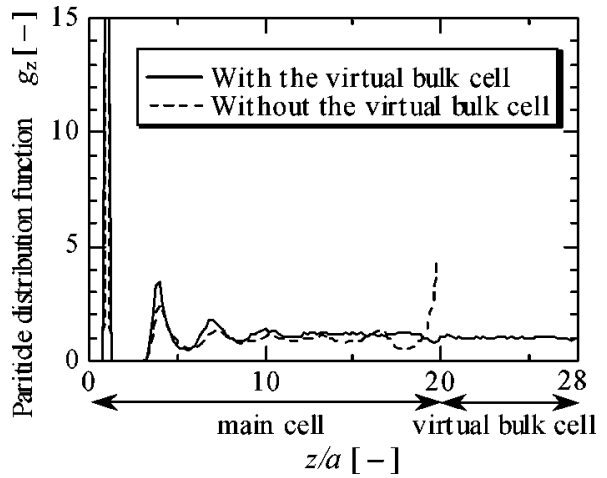

FIG. 4. A comparison of particle distribution functions $g_{z}$ between the two simulations: with and without the virtual bulk cell for $\kappa a=5, \phi=0.09$.

the bulk concentration as a result of adsorption of particles on the substrate, new particles are supplied in the reservoir region if the concentration averaged over every 100 time step is less than the desired one.

The virtual bulk cell forms a bulk phase on its own in which particle interaction follows the periodic boundary condition in all the directions. The particles in the virtual bulk cell are assumed to exert forces onto the particles in the main cell, but not to be affected by the particles in the main cell. The reason we set the virtual bulk cell is as follows: the problem of the main cell without this portion would be that the particles tend to localize in the vicinity of the top of the main cell because no interaction exists between the particles and the top of the main cell; the concentration of the particles near the top would become unrealistically high. To solve this difficulty, the virtual bulk cell is placed on the top of the main cell. Figure 4 shows the particle distribution $g_{z}$ in the $z$ direction with and without the virtual bulk cell. The distribution function $g_{z}$ is the average over the last $10^{6}$ time steps of the simulation. The major deficit in the case without the virtual bulk cell is the appearance of the unrealistic peak around the top of the main cell, which means that the particles in the main cell are gathering there if the virtual bulk cell is not mounted. On the other hand, if the main cell is equipped with this virtual cell, the $g_{z}$ for $16<z / a<20$ maintains a value almost equal to the bulk one and the particles are distributed uniformly around the top of the main cell. For this reason, it is apparent that the virtual bulk cell makes it possible to simulate the colloidal dispersion system with an adsorptive substrate under a specified bulk concentration.

\section{Simulation details}

The side length of the simulation cell was varied in the range of $30 a$ to $88 a$ depending on the thickness of the electric double layer so that at least $10 \times 10$ particles were able to adsorb onto the substrate to avoid the major effects of the periodic boundary conditions and the shape of the simulation cell. To confirm that the box size was large enough, we carried out simulations with twice the side lengths for various values of $\kappa a$, and no significant difference of structural results was found. The height of the main cell was set to be $20 a$, which would be sufficient for particles near the top of the main cell not to be affected from the adsorbed layer, as
TABLE I. Simulation parameters.

\begin{tabular}{|c|c|c|c|c|c|}
\hline$\kappa a[-]$ & 1 & 2 & 5 & 10 & 20 \\
\hline$L / a[-]$ & 88 & 66 & 44 & 36 & 30 \\
\hline$H_{\mathrm{v}} / a[-]$ & 24 & 14 & 8 & 6 & 5 \\
\hline$H_{\mathrm{m}} / a[-]$ & \multirow{2}{*}{\multicolumn{5}{|c|}{$\begin{array}{c}20 \\
8.0 \times 10^{-4}-2.8 \times 10^{-1}\end{array}$}} \\
\hline$\phi[\mathrm{vol} / \mathrm{vol}]$ & & & & & \\
\hline$\Delta t(\mathrm{~ns})$ & \multicolumn{5}{|c|}{50} \\
\hline
\end{tabular}

seen in Fig. 4 (simulations with the height $30 a$ yielded no marked differences). The cutoff distance of the interparticle potential was set as half the height of the virtual bulk cell, which was considered to be sufficient to express the particles' behavior in the bulk dispersion phase.

The structures of the adsorbed particles on the substrate were examined by changing the following parameters: $\kappa a$ and $\phi$, the volume fraction of the particles in the bulk phase. The solvent, the materials of the particles, and the substrate were modeled on water, polystyrene latex with positive surface charge, and mica, respectively. The dimensionless surface potential of the particle $\psi_{\mathrm{p}}$ was calculated with the following equation ${ }^{25}$ which relates $\psi_{\mathrm{p}}$ with the surface charge density $\sigma_{\mathrm{p}}$ assuming the constant charge condition:

$$
\frac{e \sigma_{\mathrm{p}}}{\kappa \epsilon \epsilon_{0} k_{\mathrm{B}} T}=\psi_{\mathrm{p}}+\frac{\psi_{\mathrm{p}}}{\kappa a}-\frac{\tau_{1}^{2} \kappa a}{\tau_{2}-\tau_{1} \kappa a},
$$

where

$$
\tau_{1}=2 \sinh \left(\frac{\psi_{\mathrm{p}}}{2}\right)-\psi_{\mathrm{p}}, \quad \tau_{2}=4 \tanh \left(\frac{\psi_{\mathrm{p}}}{4}\right)-\psi_{\mathrm{p}} .
$$

The calculated surface potential varied depending on the salt concentration or the thickness of the electric double layer. For instance, $\psi_{\mathrm{p}}$ was about $100 \mathrm{mV}$ when $\kappa a=10$. The simulation parameters and the physical properties used in the present study are summarized in Table I and Table II, respectively.

The initial configuration of the particles was made up at random so as to have the desired concentration; then the system was equilibrated without the adsorptive substrate for $10^{5}$ time steps, applying the periodic boundary condition in all directions. After equilibration of the system, the adsorptive substrate was set at the bottom of the main cell, and the main simulation was carried out until the system came to a stable state, i.e., the surface coverage stayed constant.

TABLE II. Physical parameters.

\begin{tabular}{lcc}
\hline \hline$a$ & $(\mathrm{~nm})$ & 50 \\
$\sigma_{\mathrm{p}}$ & $\left(\mathrm{mC} / \mathrm{m}^{2}\right)$ & 27.4 \\
$\psi_{\mathrm{s}}$ & $(\mathrm{mV})$ & -100 \\
$D$ & $\left(\mathrm{~m}^{2} / \mathrm{s}\right)$ & $4.88 \times 10^{-12}$ \\
$A_{\mathrm{pp}}$ & $(\mathrm{J})$ & $0.95 \times 10^{-20}$ \\
$A_{\mathrm{ps}}$ & $(\mathrm{J})$ & $1.60 \times 10^{-20}$ \\
$T$ & $(\mathrm{~K})$ & 298 \\
$\eta$ & $(\mathrm{Pa} \cdot \mathrm{s})$ & $8.94 \times 10^{-4}$ \\
$\epsilon$ & {$[-]$} & 78.3 \\
\hline \hline
\end{tabular}




\section{E. Data analysis}

As demonstrated later, the snapshots of adsorbed particles in the simulation would be useful information to decide whether the structures are ordered or not. There may be, however, subtle conditions in between the ordered and disordered state. A more quantitative decision becomes possible if the pair correlation function $g(r)$ and the static structure factor $S(k)$ are considered.

The pair correlation function $g(r)$ is expressed by the following equation:

$$
g(r)=\frac{1}{2 \pi r \Delta r} \frac{S}{N_{\mathrm{s}}\left(N_{\mathrm{s}}-1\right)}\left\langle\sum_{i=1}^{N_{\mathrm{s}}} \Delta N_{i}(r)\right\rangle,
$$

where $\Delta r$ is the thickness of the annulus, $N_{\mathrm{s}}$ is the number of the particles adsorbed on the substrate, $S$ is the area of the substrate, and $\Delta N_{i}(r)$ is the number of particles at a distance between $r-\Delta r / 2$ and $r+\Delta r / 2$ from a reference particle. The pair correlation functions are averaged over 100 ensembles sampled every $10^{4}$ time step from the configuration data.

The static structure factor $S(k)$, which is obtained by the Fourier transformation of the pair correlation function, was thought to be a useful criterion for freezing in a twodimensional system when the height of the first peak $S\left(k_{0}\right)$ is higher than 4.4-5.0, as reported by Ranganathan and Pathak, ${ }^{26}$ Caillol et al.,${ }^{27}$ and Broughton et al. ${ }^{28}$ Given that the structure at the freezing state corresponds to the hexagonal structure, $S(k)$ can be used for judging whether or not structures are ordered. In the present study, the criterion of the height of the first peak for an ordered state is set to be 4.7 , which is the middle value between 4.4 and 5.0, reported as above.

\section{RESULTS AND DISCUSSION}

\section{A. Formation of ordered structures}

In this section, we examine the effect of salt concentration and particle concentration in bulk onto the orderdisorder transition of the adsorbed particle phase, to clarify the bulk condition needed for the order formation.

\section{Effect of salt concentration}

Time evolution of surface coverage $\theta$ for five different values of $\kappa a$ under $\phi=1.0 \times 10^{-2}$ is shown in Fig. 5, while the snapshots at the end of the simulation, the pair correlation functions $g(r)$, and the static structure factors $S(k)$ are shown in Figs. 6(a)-6(e). Note that no desorption of particles was observed because of the strong attraction between the particles and the substrate under the simulation conditions employed in the present study; the adsorption process is essentially irreversible. It is seen from Fig. 5 that the surface coverage for $\kappa a=1$ and 2 increases with time rapidly and then reaches a certain value, after which it remains almost constant; the surface coverage for larger $\kappa a$ goes gradually up to a plateau value, and the final surface coverage is greater for a larger value of $\kappa a$. The above variation in the adsorption rate and surface coverage against salt concentration is thought to be natural in the light of the interparticle interaction. For a lower salt concentration, the interaction forces become strong and long-ranged. Then the strong

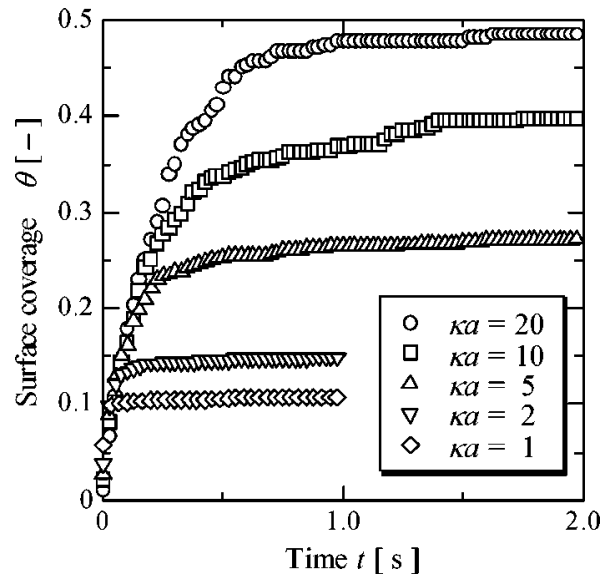

FIG. 5. Time evolution of the surface coverage for five values of $\kappa a$ at $\phi=0.01$.

particle-substrate attraction makes it easier for the bulk particles to adsorb onto the substrate. At the same time a large exclusive area of a particle due to the strong particle-particle repulsion leads to low surface coverage.

The structures of adsorbed particles on the substrate for $\kappa a=1$ and 2 can be concluded to attain an ordered state because $g(r)$ exhibits a split in the second peak and the first peak of $S(k)$ exceeds 4.7, as shown in Figs. 6(a) and 6(b). On the other hand, the structures for larger $\kappa a$ are disordered, as shown by $g(r)$ of a liquidlike state and lower first peaks of $S(k)$ around 2.5 in Figs. 6(c)-6(e). These results indicate that salt concentration affects the order-disorder transition of the structure on the substrate as well as the adsorption rate: ordered structures can be formed more easily for small $\kappa a$ than for large $\kappa a$.

\section{Effect of bulk concentration}

We performed simulations varying the volume fraction of particles $\phi$ for the following $\kappa a: 1,2,5,10$, and 20. As a typical example of the results, adsorption kinetics with $\kappa a$ $=5$ are shown in Fig. 7, in which the final plateau values of surface coverage $\theta_{\mathrm{f}}$ depend evidently on $\phi$. Compared with the irreversible monolayer adsorption of molecules, which would show a constant amount of adsorbed molecules regardless of the bulk concentration, this dependence on $\phi$ in the colloidal adsorption is peculiar. As given in a detailed explanation in Refs. 21 and 22, this behavior would be attributed to an energy barrier created by the electrostatic repulsion between adsorbed particles and bulk particles, and the existence of the energy barrier could be verified, though indirectly, by a depletion region near the substrate in Fig. 4 (Fig. 9 in Ref. 21). The analysis of the structure based on $g(r)$ and $S(k)$ clarified that there exists a boundary for order formation between $\phi=0.06$ and $\phi=0.08$. Quite naturally, the final coverage for $\phi=0.08$ is larger than that for $\phi=0.06$. The difference of the two structures can be simply explained by the following: with increasing surface coverage, the disordered arrangement of particles with various interparticle distances would produce some portions of high-energy interactions, and then the ordered structure with an almost uniform interparticle distance becomes more stable. To make the 

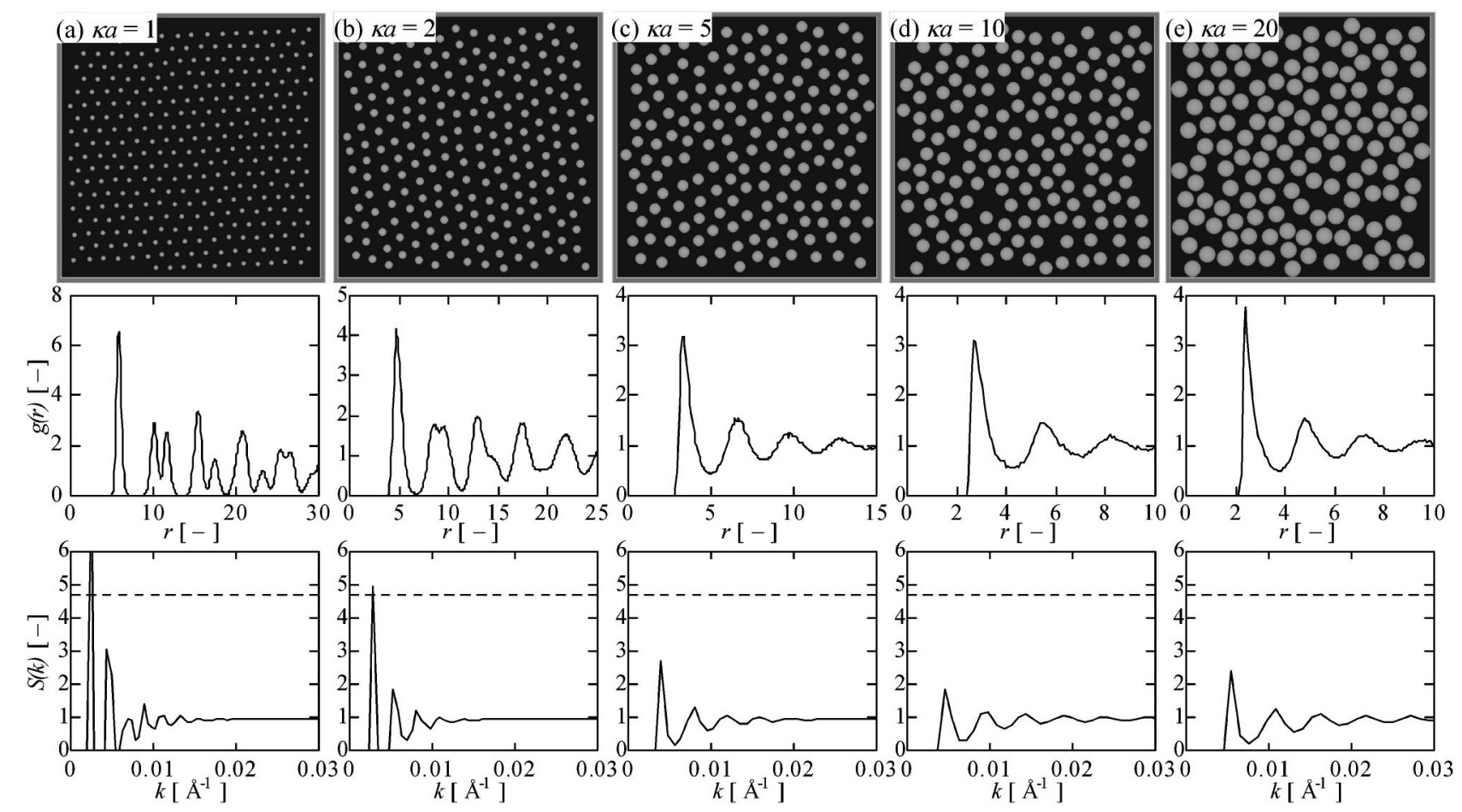

FIG. 6. Snapshots at the end of the simulation, the pair correlation functions, and the static structure factors at $\phi=0.01$ for (a) $\kappa a=1$, (b) $\kappa a=2$, (c) $\kappa a$ $=5$, (d) $\kappa a=10$, and (e) $\kappa a=20$. The dotted line for the static structure factor denotes $S(k)=4.7$.

order-disorder boundary clear, we investigated the timeevolution of $g(r)$ and $S(k)$ and specified the critical coverage $\theta^{\mathrm{c}}$ for order formation as the lowest coverage to form ordered structures. The critical coverage $\theta^{\mathrm{c}}$ for each $\kappa a$ is summarized in Table III.

The peculiar behavior would also be attributed to the limited simulation time, and then we investigated infinitetime coverage $\theta_{\infty}$ using the extrapolation method by Schaaf et $a .^{29}$ according to the asymptotic power law,

$$
\theta_{\infty}-\theta(t) \sim t^{-2 / 3} \text {. }
$$

As demonstrated in Fig. 8, which is the recast of Fig. 7, the dependence of the infinite-time coverage $\theta_{\infty}$ on bulk concentration is less than that of the final coverage from the simulation. However, the difference in $\theta_{\infty}$ would be important

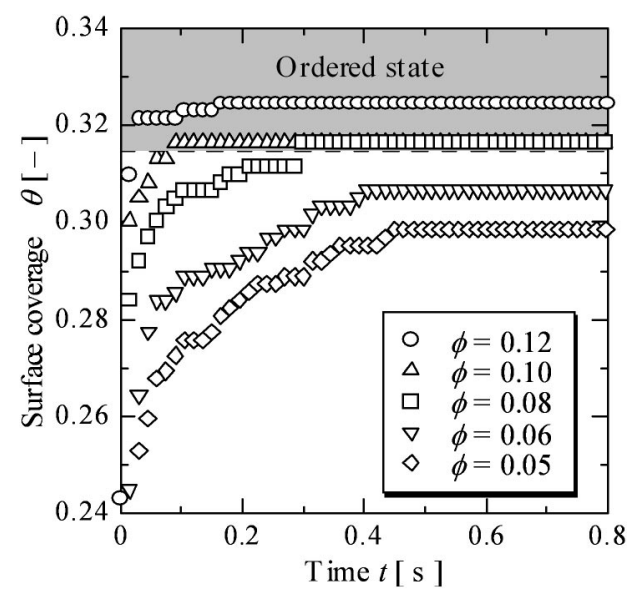

FIG. 7. Time evolution of surface coverage for various $\phi$ at $\kappa a=5$. because that for $\phi=0.06$ is smaller than the critical coverage $\theta^{\mathrm{c}}$ while it is larger for $\phi=0.08$ : order or disorder of adsorbed structures seems to be dependent on bulk concentration.

In the same manner, we estimated the extrapolated infinite-time coverage $\theta_{\infty}$ for other values of $\kappa a$, and the obtained $\theta_{\infty}$ normalized by the critical surface coverage $\theta^{\mathrm{c}}$ are plotted against the bulk concentration for five different values of $\kappa a$ in Fig. 9. The normalized surface coverage $\theta_{\infty} / \theta^{\mathrm{c}}$ is dependent on bulk concentration, and especially for large $\kappa a$, the dependency is quite significant. Figure 9 also demonstrates that the bulk concentration required to reach an ordered state $\left(\theta_{\infty} / \theta^{\mathrm{c}} \geqslant 1.0\right)$ remarkably increases with increasing $\kappa a$, where these high bulk concentrations for large $\kappa a$ are thought to serve to compensate the decrease in the bulk potential associated with the increase of $\kappa a$.

Here it should be noted that these results were obtained with no hydrodynamic interactions between particles and between a particle and the substrate. However, this would not significantly affect the adsorbed amount of particles because

TABLE III. Critical values of ordered states for various $\kappa a$ together with the hard disk data of Alder and Wainwright ${ }^{32}$

\begin{tabular}{lcccccc}
\hline \hline$\kappa a[-]$ & 1 & 2 & 5 & 10 & 20 & Hard disk \\
\hline$\theta^{\mathrm{c}}[-]$ & $0.787 \times 10^{-1}$ & 0.156 & 0.315 & 0.456 & 0.576 & 0.72 \\
$a_{\mathrm{eff}}^{\mathrm{c}}(\mathrm{nm})$ & 166 & 121 & 85 & 71 & 62 & $\ldots$ \\
$E_{\mathrm{av}}^{\mathrm{c}}\left[k_{\mathrm{B}} T\right]$ & 16 & 8.2 & 2.5 & 1.7 & 0.6 & $\ldots$ \\
$P^{\mathrm{c} S}$ & 31.5 & 21.8 & 15.3 & 13.1 & 12.7 & 9.64 \\
$N_{\mathrm{S}} k_{\mathrm{B}} T$ & & & & & & \\
\hline \hline
\end{tabular}




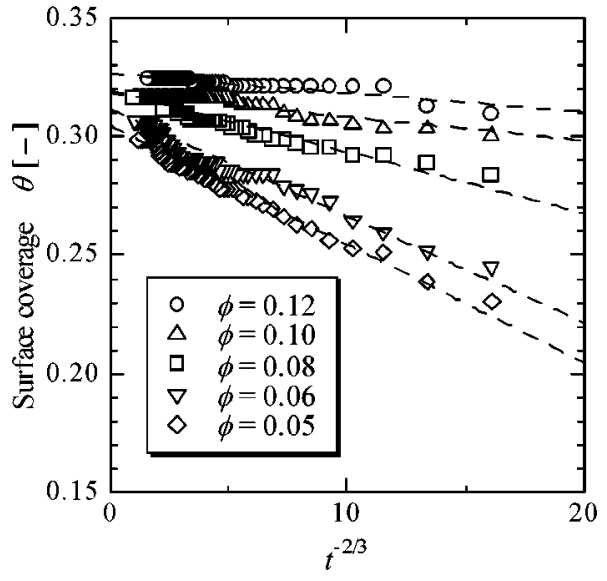

FIG. 8. The extrapolation of the results in Fig. 7 following the asymptotic power law of $t^{-2 / 3}$

the above discussion suggests that the particle adsorption is kinetically limited by the energy barrier: hydrodynamic interactions between particles do not prevail, as mentioned earlier, and hydrodynamic drag forces due to the substrate would act on an adsorbing particle only after it overcomes the energy barrier.

\section{B. Determinant factor for order formation}

As for the crystallization transition of charge-stabilized colloidal dispersions, the simulation study of Stevens and Robbins, ${ }^{30}$ examining the effect of shear on the solid-liquid boundary, revealed that the nonequilibrium phase diagram shows considerable commonality to the bcc and fcc systems. This kind of the commonality of the order-disorder boundary is also expected in the two-dimensional structures. In this section, taking notice of the commonality to $\kappa a$, we examine physical properties of the adsorbed phase and their critical values at the order-disorder transition. As mentioned above, surface coverage was one of the factors that influence the transition, but appeared not to have a common critical value for various $\kappa a$; we therefore examine other properties of the adsorbed structures and try to find out what is the determinant factor, which is common to any value of $\kappa a$, for order formation.

\section{The difference of structures for different $\kappa$}

Before discussing the determinant factor common to any $\kappa a$, we examine the structure difference due to the change of $\kappa a$. The snapshots of the ordered structures for different $\kappa a$ are shown in Figs. 10(a)-10(e). All the structures in Fig. 10 are in an ordered state, but the characteristic length of the structures is quite different, reflecting the difference of the exclusive area around a particle due to the particle-particle repulsive interaction. The effective radii including the exclusive area, which are estimated from $g(r)$ of the ordered structure as half of the nearest particle-particle distance, are summarized in Table III. The effective radius decreases with increasing $\kappa a$, which indicates that salt concentration can control the periodicity of the ordered structures.

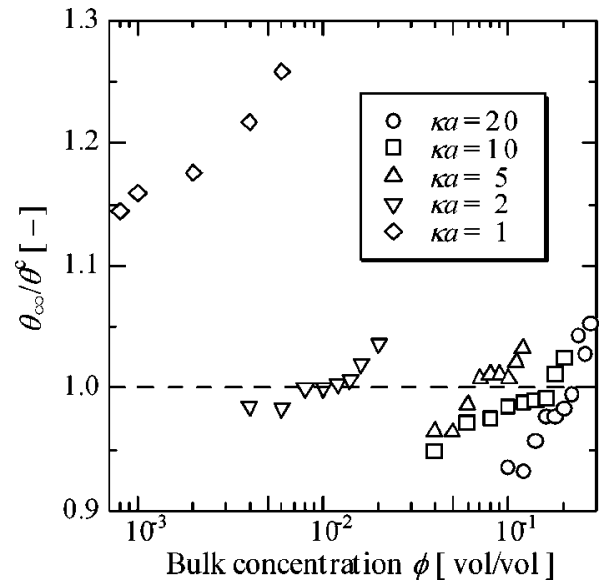

FIG. 9. Extrapolated coverage against the bulk concentration for five different values of $\kappa a$.

\section{Average potential of the adsorbed particles}

First, we consider an average potential of an adsorbed particle due to the interactions between the adsorbed particles. The average potential $E_{\text {av }}$ was calculated as the sum of the potential acting on a particle, and the average over all particles and over many ensembles was then taken:

$$
E_{\mathrm{av}}=\frac{1}{N_{\mathrm{s}}}\left\langle\sum_{i=1}^{N_{\mathrm{s}}} \sum_{\substack{j=1 \\ j \neq i}}^{N_{\mathrm{s}}} E_{\mathrm{pp}}\left(r_{i j}\right)\right\rangle,
$$

where $r_{i j}$ is the distance between adsorbed particles $i$ and $j$, and $E_{\mathrm{pp}}$ is the sum of the electrostatic potential $E_{\mathrm{el}(\mathrm{pp})}$ and the van der Waals potential $E_{\mathrm{vdW}(\mathrm{pp})}$. Considering that the potential of a particle has been sometimes used for predicting an effective radius of a particle (discussed later), the average potential would be a likely candidate for the determinant factor. If the average potential is the determinant factor for order formation, an ordered structure would appear when the average potential exceeds a certain value common to different values of $\kappa a$.

The critical average potentials between the order and disorder state, $E_{\mathrm{av}}^{\mathrm{c}}$, are summarized in Table III. The critical average potential $E_{\mathrm{av}}^{\mathrm{c}}$ corresponds to the average potential of the structures with the critical surface coverage $\theta^{\mathrm{c}}$. It shows, contrary to the expectation, that the critical average potentials for order formation remarkably increase with decreasing $\kappa a$. Consequently, the average potential is proved not to be the determinant factor for the order formation.

\section{Two-dimensional pressure on the substrate}

Second, we examine the two-dimensional pressure of the adsorbed particles owing to the interparticle interaction. The two-dimensional pressure $P_{\mathrm{s}}$ is given by the following equation according to the virial theorem: ${ }^{31}$

$$
\frac{P_{\mathrm{s}} S}{N_{\mathrm{s}} k_{\mathrm{B}} T}=1-\frac{1}{2 N_{\mathrm{s}} k_{\mathrm{B}} T}\left\langle\sum_{i=1}^{N_{\mathrm{s}}} \sum_{j>i}^{N_{\mathrm{s}}} r_{i j} \frac{\mathrm{d} E_{\mathrm{pp}}\left(r_{i j}\right)}{\mathrm{d} r_{i j}}\right\rangle .
$$

In the same manner as that for the average potential, the critical two-dimensional pressures $P^{\mathrm{c}}$ for different $\kappa a$ were calculated, which are shown in Table III. Similarly to the 

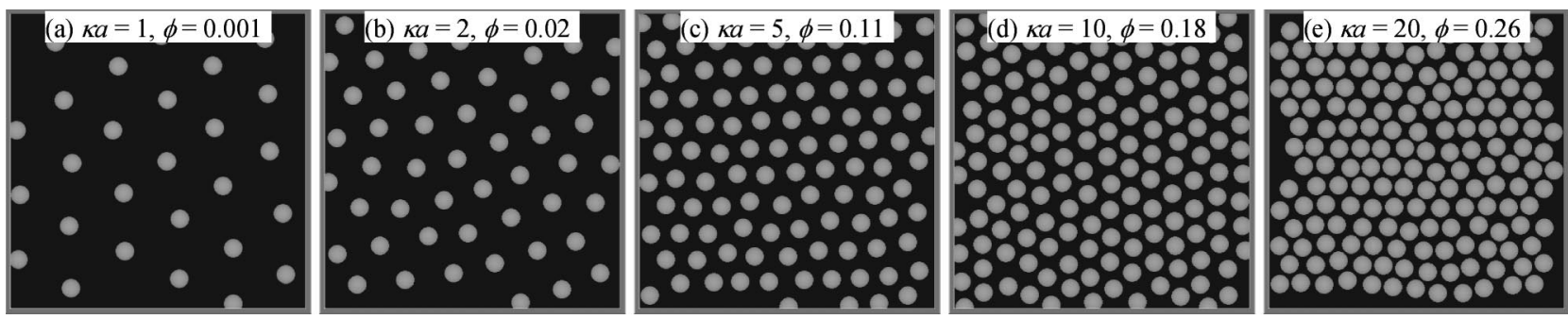

FIG. 10. Snapshots of ordered structures for (a) $\kappa a=1, \phi=0.001$, (b) $\kappa a=2, \phi=0.02$, (c) $\kappa a=5, \phi=0.11$, (d) $\kappa a=10, \phi=0.18$, and (e) $\kappa a=20, \phi=0.26$. The side length of each snapshot is $1.5 \mu \mathrm{m}$.

case of the average potential, the border values needed to form the ordered structure are different for each $\kappa a$. Thus the two-dimensional pressure is not the determinant factor. On the other hand, Table III also shows that higher pressure is required to form ordered structures for smaller $\kappa a$, and that as $\kappa a$ increases, the values of $\theta^{\mathrm{c}}$ and $P^{\mathrm{c}}$ are likely to converge to that of the two-dimensional hard-disk system obtained from the simulation by Alder and Wainwright. ${ }^{32}$ The tendency - the smaller the value of $\kappa a$ the higher the pressure-is consistent with the experimental results of Takano and Hachisu, ${ }^{33}$ who experimentally demonstrated a phase transition from disordered to ordered state in a colloidal dispersion and concluded that the phase transition must be of a Kirkwood-Alder transition type on the basis of the comparison of the transition pressure obtained from their experiment with that from the simulation result by Alder et al. $^{34}$ It would thus be reasonable to consider the orderdisorder transition of adsorbed structures on a substrate as the Kirkwood-Alder transition type as well as that of colloidal dispersions, taking account of the qualitative accordance of the tendency of the pressure.

The above discussion, therefore, reveals that the orderdisorder transition of the structure formed by the adsorbed particles must be of the Kirkwood-Alder transition type, though the two-dimensional pressure is found not to be the determinant factor for the order formation common to various $\kappa a$.

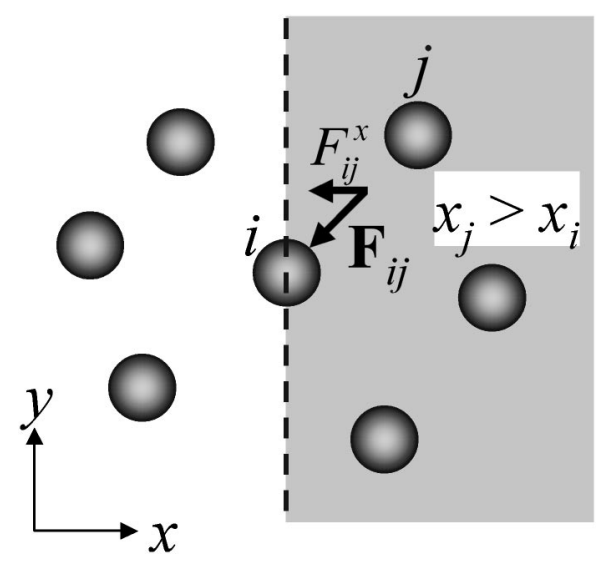

FIG. 11. A schematic for the calculation of the one-directional force of an adsorbed particle $i$.

\section{One-directional average force}

Then, the "force" of an adsorbed particle would be worth discussing because it seems reasonable that the configuration of adsorbed particles is affected by forces acting among adsorbed particles, and we accordingly define the one-directional average force acting on an adsorbed particle, $F_{\text {av }}$. Technically the sample of the force is calculated as follows. First $F_{x+}^{i}$ is figured out as the sum of the $x$-component forces on particle $i$ acting from particles $j$ existing on the right-hand side of it, i.e., satisfying $x_{j}>x_{i}$ (Fig. 11 ), and is expressed by the following equation:

$$
F_{x+}^{i}=\sum_{\substack{j=1 \\ x_{j}>x_{i}}}^{N_{\mathrm{s}}} F_{i j}^{x}
$$

where $F_{i j}^{x}$ is the $x$ component of the force acting on particle $i$ from $j$. In the same manner, $F_{x-}^{i}, F_{y+}^{i}$, and $F_{y-}^{i}$ are calculated as, respectively, the $x$-components from left-hand side particles $\left(x_{j}<x_{i}\right)$, the $y$-components from particles satisfying $y_{j}>y_{i}$, and the $y$-components from particles with $y_{j}<y_{i}$. Then, averaging of the absolute values over four components, over all particles, and over many ensembles is taken as given by the following equation:

$$
F_{\mathrm{av}}=\frac{1}{N_{\mathrm{s}}}\left\langle\sum_{i=1}^{N_{\mathrm{s}}}\left(\frac{\left|F_{x+\mid}^{i}\right|+\left|F_{x-}^{i}\right|+\left|F_{y+}^{i}\right|+\left|F_{y-}^{i}\right|}{4}\right)\right\rangle .
$$

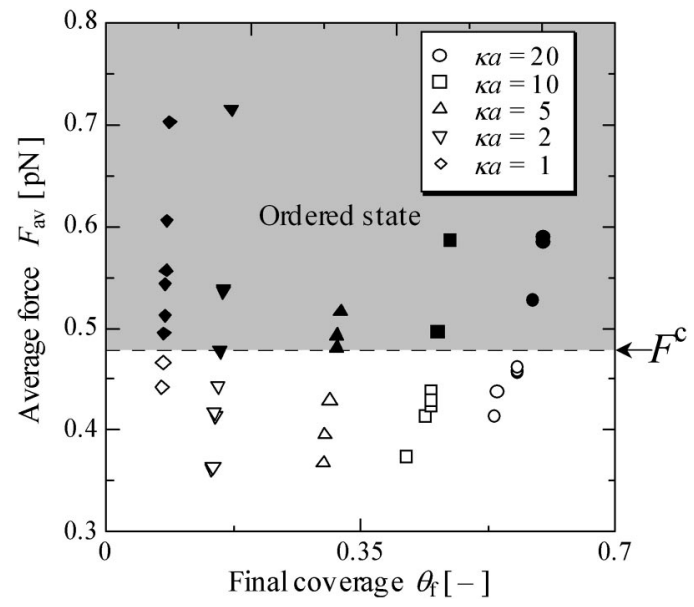

FIG. 12. The one-directional average force of an adsorbed particle as a function of final coverage for five values of $\kappa a$. Closed plots denote the formation of an ordered structure. 
As seen in Fig. 12, the average forces for five different values of $\kappa a$ exhibit a common boundary value $F^{\mathrm{c}}$ for order formation with commonality similar to what Stevens and Robbins $^{30}$ found. Consequently, the average force $F_{\text {av }}$ is found to be the determinant factor for order formation common to different $\kappa a$, which means that a structure composed of adsorbed particles on a substrate is expected to form an ordered structure only when the average force $F_{\text {av }}$ exceeds the limiting value $F^{\mathrm{c}}$. In the ordered structure the adsorbed particles must be localized at each fixed position. Then the critical force $F^{\mathrm{c}}$ is interpreted as the force needed to keep the ordered structure by limiting the particles' diffusion caused by the Brownian motion. Here it should be noted that the average force might look similar to the two-dimensional pressure (Sec. III B 2) in the sense that the both two figure out the sum of the forces, but the force summation in the pressure calculation is weighted with the interparticle distance $r$ as given in the second term of Eq. (16). Since the characteristic length of the adsorbed structures is different depending on the value of $\kappa a$, the $r$ weighting would lead to the failure of the two-dimensional pressure as the determinant factor.

Looking deep into the particle-particle interaction potential, we found that the magnitude of $F^{\mathrm{c}}$ in the order of $10^{-12} \mathrm{~N}$ corresponds to the location at which the interaction potential energy of the order of $k_{\mathrm{B}} T$ acts between the particles (the potential energy, of course, varies with $\kappa a$ to some extent, as discussed in Sec. III B 1). Because the energy that particles can normally possess is also in the order of $k_{\mathrm{B}} T$, the magnitude of the obtained $F^{\mathrm{c}}$ would be quite reasonable. It would deserve much attention that the common factor is not the potential, nor the pressure, but the "force" acting between the adsorbed particles.

\section{Estimation of critical surface coverage for order formation}

Here we propose a new approach to predict the surface coverage at the order-disorder transition by means of applying the common boundary value $F^{\mathrm{c}}$ to a force-based model, which is one of the advantages of having discovered the one-directional average force. A comparison of the proposed model with other models previously developed will be made in the following.

The critical surface coverage for order formation can be estimated by the following simple equation:

$$
\theta=\theta_{\max }\left(\frac{a}{a_{\text {eff }}}\right)^{2},
$$

where $\theta_{\max }$ is the maximum surface coverage for a hard disk. Since we are interested in the surface coverage at the orderdisorder transition, the close-packed coverage $\pi / 2 \sqrt{3}$ is appropriate as the value of $\theta_{\max }$, while $\theta_{\max }$ of 0.547 for the RSA jamming limit ${ }^{11}$ is suitable only for the case with no rearrangement of the structure of adsorbed particles. As for the effective hard-disk radius $a_{\text {eff }}$, a potential-based model and an energetically based model have been proposed.

Russel et $a .^{35}$ have claimed that the phase transition from disorder to order would occur when the interparticle

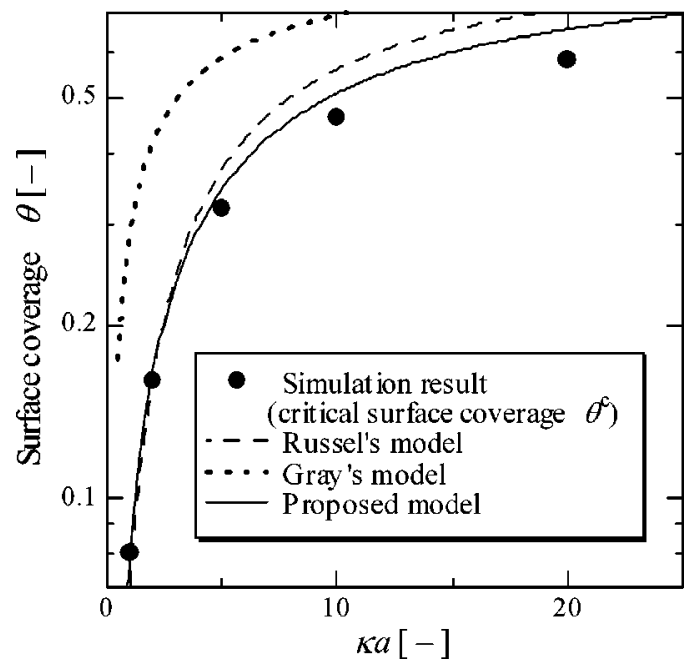

FIG. 13. A comparison of the surface coverage as a function of $\kappa a$ between models and the critical surface coverage obtained from the simulation.

interaction potential is about $1 k_{\mathrm{B}} T$ and provided an analytical approximation for the calculation of $a_{\text {eff }}$ by defining it as the radial position where the electrostatic potential is equal to $1 k_{\mathrm{B}} T$ :

$$
a_{\mathrm{eff}}=\frac{1}{2 \kappa} \ln \left(\frac{A}{\ln A}\right),
$$

with

$$
A=\left(\frac{4 \pi k T \epsilon \epsilon_{0} a}{e^{2}}\right)\left(\frac{\psi_{\mathrm{p}}+4 \gamma \Omega \kappa a}{1+\Omega \kappa a}\right)^{2} \kappa a \exp (2 \kappa a),
$$

where Eq. (5) is used as the electrostatic potential. The results of this potential-based model are compared with the critical surface coverage $\theta^{\mathrm{c}}$ in Fig. 13. On the whole, the potential-based model gives a fairly good prediction, and an adjustment in the cut-off potential may improve the estimation. ${ }^{6}$ However, as long as the constant cut-off potential is used, the potential-based model will not give a consistent estimation throughout various values of $\kappa a$ : the results of the average potential listed in Table III indicate that the border potentials for order formation cannot stay constant for various values of $\kappa a$.

More recently, Gray and Bonnecaze ${ }^{21}$ have proposed an energetically based model, including the effect of the wall potential. This model is based on the balance between the particle-substrate attractive energy and the repulsive energy between adsorbed particles that is composed of energy from a newly adsorbing particle and incremental repulsions between already adsorbed particles.

$$
E_{\mathrm{el}(\mathrm{ps})}(h=0)=\left\langle E_{\mathrm{el}(\mathrm{pp})}\right\rangle+N_{\mathrm{s}} \frac{\left.\partial\left\langle E_{\mathrm{el}(\mathrm{pp}}\right)\right\rangle}{\partial N_{\mathrm{s}}},
$$

where $E_{\mathrm{el}(\mathrm{ps})}(h=0)$ is the binding energy of an adsorbed particle with the substrate, and $\left.\left\langle E_{\mathrm{el}(\mathrm{pp}}\right)\right\rangle$ is the average interparticle energy per particle. Substitution of the particleparticle and particle-substrate potentials of Eqs. (5) and (6) yields 


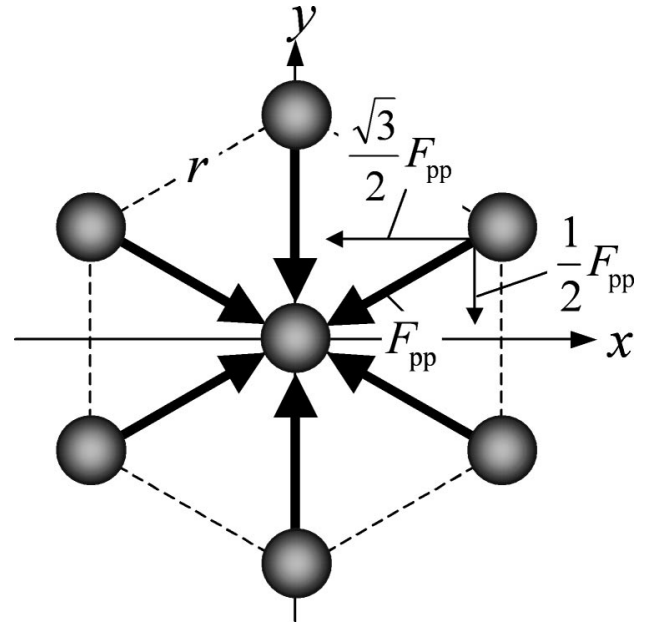

FIG. 14. A schematic for the calculation of the average force of a particle placed at the center of a perfect hexagonal lattice.

$$
\begin{aligned}
\frac{1}{4} n\left(\frac{\psi_{\mathrm{p}}+4 \gamma \Omega \kappa a}{1+\Omega \kappa a}\right) \exp [-\kappa a(r-2)]\left(\frac{3}{r}+\kappa a\right) \\
+\tanh \left(\frac{\psi_{\mathrm{s}}}{4}\right)=0
\end{aligned}
$$

where $n$ is the coordination number of adsorbed particles and $n=6$ when a hexagonal arrangement is assumed. We can obtain $a_{\text {eff }}$ as half of the average nearest-neighbor distance $r$ satisfying Eq. (23). The result of Gray's model is also shown in Fig. 13. As seen, this model highly overestimates surface coverage for all values of $\kappa a$ because, as the authors mentioned, this model reflects the equilibrium state though the adsorption system employed is kinetically limited by the energy barrier formed by the adsorbed particles.

The force-based model we propose here defines $a_{\text {eff }}$ as the radial position where the one-directional average force is equal to the critical value, $F^{\mathrm{c}}$. Assuming a perfect hexagonal lattice with interparticle distance $r$ as in Fig. 14, the average force acting on a particle placed at the center of the hexagon, $F_{\mathrm{av}}^{\mathrm{hex}}$, is simply given by Eq. (24) since $\left|F_{x+}^{i}\right|=\left|F_{x-}^{i}\right|$ $=(\sqrt{3} / 2) F_{\mathrm{pp}} \times 2,\left|F_{y+}^{i}\right|=\left|F_{y-}^{i}\right|=F_{\mathrm{pp}}+(1 / 2) F_{\mathrm{pp}} \times 2$,

$$
F_{\mathrm{av}}^{\mathrm{hex}}=\frac{F_{\mathrm{pp}} \times 2+\frac{1}{2} F_{\mathrm{pp}} \times 4+\frac{\sqrt{3}}{2} F_{\mathrm{pp}} \times 4}{4}=\frac{2+\sqrt{3}}{2} F_{\mathrm{pp}},
$$

where $F_{\mathrm{pp}}$ is the force composed of the electrostatic and van der Waals forces acting between two particles. To be exact, $F_{\mathrm{av}}^{\mathrm{hex}}$ is dependent on the orientation of the structure relative to the $x, y$ axis, but the dependency was found to be smaller than $\pm 2 \%$, which would be negligible. Then the effective radius $a_{\text {eff }}(=r / 2)$ can be obtained by the trial calculation of the distance $r$ between particles satisfying the equation $F_{\mathrm{av}}^{\text {hex }}$ $=F^{c}$. More practically, $a_{\text {eff }}$ can be derived by the following analytic form similar to Eq. (20), under the assumption that the van der Waals force is negligible in comparison with the electrostatic force,

$$
\begin{aligned}
a_{\mathrm{eff}}= & \frac{1}{2 \kappa} \ln \left[\frac{B}{\left\{\ln \left(\frac{B}{(\ln 2 B)^{2}}+\frac{B}{\ln 2 B}\right)\right\}^{2}}\right. \\
& \left.+\frac{B}{\ln \left(\frac{B}{(\ln 2 B)^{2}}+\frac{B}{\ln 2 B}\right)}\right],
\end{aligned}
$$

with

$$
\begin{aligned}
B= & \frac{2+\sqrt{3}}{2} \frac{1}{F^{\mathrm{c}}}\left(\frac{4 \pi k_{\mathrm{B}} T \epsilon \epsilon_{0}}{e^{2}}\right) \\
& \times\left(\frac{\psi_{\mathrm{p}}+4 \gamma \Omega \kappa a}{1+\Omega \kappa a}\right)^{2}(\kappa a)^{2} \exp (2 \kappa a) .
\end{aligned}
$$

Equation (25) holds true especially for $\kappa a \leqslant 10$ because of the assumption, but even for $\kappa a=20$, the difference between $a_{\text {eff }}$ from the trial calculation (considering the van der Waals force) and from Eq. (25) turned out to be about $0.5 \%$. The result of our model is given also in Fig. 13. The surface coverage estimated by the proposed model gives better agreement throughout all values of $\kappa a$ than those of other models. What is more, the advantage of our model lies in the applicability to the system with adhesive forces or frictional forces because this model is based on the force acting on an adsorbed particle.

The force-based model is capable of predicting the surface coverage at the order-disorder transition, but includes only two-dimensional information. The bulk concentration or the time needed to reach ordered structures is also important as well as the surface coverage, and should be predicted by an analytical model. To do this, the incorporation of the adsorption kinetics into the model would be essential and could be done by extending the force-based model. Work along this line is under progress.

\section{CONCLUSIONS}

A three-dimensional simulation model for a colloidal dispersion system with an adsorptive substrate under a specified bulk concentration is proposed. Through simulating colloidal adsorption under various conditions and examining the condition for order formation, the following conclusions are drawn.

(1) There exists critical surface coverage required to form an ordered structure, and extrapolated infinite-time coverage seems to depend on bulk concentration especially for large $\kappa a$ probably because the decrease in the bulk potential must be compensated by the increase of bulk concentration. Also, the order-disorder transition of adsorbed structures is considered to be dependent on bulk concentration.

(2) The phase transition of the adsorbed particles on a substrate from a disordered state to an ordered state appears to be of the Kirkwood-Alder type through the examination of the two-dimensional pressure.

(3) The determinant factor for the order formation is found to be what we call a "one-directional average force" 
acting on an adsorbed particle, which stays constant for all values of $\kappa a$ examined. The ordered structures are formed when this force exceeds a certain limiting value, which presumably corresponds to a force needed to have adsorbed particles fixed at a position against the thermal Brownian motion.

(4) A new model to estimate the surface coverage at the order-disorder transition is proposed, utilizing the limiting value of the one-directional average force. A comparison with simulation results demonstrated that this model gives a better estimation than the other models.

The present simulation model assumes a flat and smooth substrate on which the adsorbed particles move freely. On the other hand, a system with no surface diffusion will never attain an ordered structure. Since some experimental results $^{5-10}$ show possibilities of order formation, the property of a substrate would be in between the two conditions of free surface diffusion and no surface diffusion. The simulation should thus include frictional forces or adhesive forces to examine these intermediate systems, which is under investigation.

${ }^{1}$ H. Hattori, Adv. Mater. (Weinheim, Ger.) 13, 51 (2001).

${ }^{2}$ J. D. Joannopoulos, R. D. Meade, and J. N. Winn, Photonic Crystals (Princeton University Press, Princeton, 1995).

${ }^{3}$ K. Muramatsu, M. Takahashi, K. Tajima, and K. Kobayashi, J. Colloid Interface Sci. 242, 127 (2001).

${ }^{4}$ A. S. Dimitrov and K. Nagayama, Langmuir 12, 1303 (1996).

${ }^{5}$ C. A. Johnson and A. M. Lenhoff, J. Colloid Interface Sci. 179, 587 (1996).

${ }^{6}$ M. Semmler, E. K. Mann, J. Rička, and M. Borkovec, Langmuir 14, 5127 (1998).

${ }^{7}$ M. Semmler, E. K. Mann, J. Rička, and M. Borkovec, Colloids Surf., A 165, 79 (2000).

${ }^{8}$ D. A. Antelmi and O. Spalla, Langmuir 15, 7478 (1999).

${ }^{9}$ K. Yamaguchi, T. Taniguchi, S. Kawaguchi, and K. Nagai, Chem. Lett. 7, 658 (2001).
${ }^{10}$ K. Yamaguchi, T. Taniguchi, S. Kawaguchi, and K. Nagai, Colloid Polym. Sci. 280, 942 (2002).

${ }^{11}$ J. Feder, J. Theor. Biol. 87, 237 (1980).

${ }^{12}$ Z. Adamczyk, M. Zembala, B. Siwek, and P. Warszyński, J. Colloid Interface Sci. 140, 123 (1990).

${ }^{13}$ Z. Adamczyk, B. Siwek, M. Zembala, and P. Belouschek, Adv. Colloid Interface Sci. 48, 151 (1994).

${ }^{14}$ Z. Adamczyk and P. Warszyński, Adv. Colloid Interface Sci. 63, 41 (1996).

${ }^{15}$ I. Pagonabarraga et al., J. Chem. Phys. 105, 7815 (1996).

${ }^{16}$ Z. Adamczyk, B. Siwek, M. Zembala, and P. Weroński, J. Colloid Interface Sci. 185, 236 (1997)

${ }^{17}$ M. R. Oberholzer et al., J. Colloid Interface Sci. 194, 138 (1997).

${ }^{18}$ Z. Adamczyk, P. Weroński, and E. Musiał, J. Colloid Interface Sci. 241, 63 (2001).

${ }^{19}$ J. J. Gray, D. H. Klein, B. A. Korgel, and R. T. Bonnecaze, Langmuir 17, 2317 (2001).

${ }^{20}$ M. R. Oberholzer, N. J. Wagner, and A. M. Lenhoff, J. Chem. Phys. 107, 9157 (1997).

${ }^{21}$ J. J. Gray and R. T. Bonnecaze, J. Chem. Phys. 114, 1366 (2001).

${ }^{22}$ J. J. Gray and R. T. Bonnecaze, Langmuir 17, 7935 (2001).

${ }^{23}$ E. J. W. Verwey and J. Th. G. Overbeek, Theory of The Stability of Lyophobic Colloids (Elsevier, Amsterdam, 1948).

${ }^{24}$ D. L. Ermak, J. Chem. Phys. 62, 4189 (1975).

${ }^{25}$ J. E. Sader, J. Colloid Interface Sci. 188, 508 (1997).

${ }^{26}$ S. Ranganathan and K. N. Pathak, Phys. Rev. A 45, 5789 (1992).

${ }^{27}$ J. M. Caillol, D. Levesque, J. J. Weis, and J. P. Hansen, J. Stat. Phys. 28, 325 (1982).

${ }^{28}$ J. Q. Broughton, G. H. Gilmer, and J. D. Weeks, Phys. Rev. B 25, 4651 (1982).

${ }^{29}$ P. Schaaf, A. Johner, and J. Talbot, Phys. Rev. Lett. 66, 1603 (1991).

${ }^{30}$ M. J. Stevens and M. O. Robbins, Phys. Rev. E 48, 3778 (1993).

${ }^{31}$ M. P. Allen and D. J. Tildesley, Computer Simulation of Liquids (Oxford University Press, Oxford, 1987).

${ }^{32}$ B. J. Alder and T. E. Wainwright, Phys. Rev. 127, 359 (1962).

${ }^{33}$ K. Takano and S. Hachisu, J. Chem. Phys. 67, 2604 (1977).

${ }^{34}$ B. J. Alder, W. G. Hoover, and D. A. Young, J. Chem. Phys. 49, 3688 (1968).

${ }^{35}$ W. B. Russel, D. A. Saville, and W. R. Schowalter, Colloidal Dispersions (Cambridge University Press, Cambridge, 1989). 